\title{
Mechanism of decreased sensitivity of dobutamine associated left ventricular wall motion analyses for appreciating inducible ischemia in older adults
}

Sujethra Vasu', William C Little², Timothy M Morgan ${ }^{3}$, Richard B Stacey ${ }^{1}$, William O Ntim ${ }^{4}$, Craig Hamilton, Vinay Thohan ${ }^{6}$, Caroline Chiles $^{7}$ and William Gregory Hundley ${ }^{1,7^{*}}$

\begin{abstract}
Background: Dobutamine associated left ventricular (LV) wall motion analyses exhibit reduced sensitivity for detecting inducible ischemia in individuals with increased LV wall thickness. This study was performed to better understand the mechanism of this reduced sensitivity in the elderly who often manifest increased LV wall thickness and risk factors for coronary artery disease.

Methods: During dobutamine cardiovascular magnetic resonance (DCMR) stress testing, we assessed rate pressure product (RPP), aortic pulse wave velocity (PWV), LV myocardial oxygen demand (pressure volume area, PVA, mass, volumes, concentricity, and the presence of wall motion abnormalities (WMA) and first pass gadolinium enhanced perfusion defects (PDs) indicative of ischemia in 278 consecutively recruited individuals aged $69 \pm 8$ years with pre-existing or known risk factors for coronary artery disease. Each variable was assessed independently by personnel blinded to participant identifiers and analyses of other DCMR or hemodynamic variables.
\end{abstract}

Results: Participants were $80 \%$ white, $90 \%$ hypertensive, $43 \%$ diabetic and $55 \%$ men. With dobutamine, $60 \%$ of the participants who exhibited PDs had no inducible WMA. Among these participants, myocardial oxygen demand was lower than that observed in those who had both wall motion and perfusion abnormalities suggestive of ischemia $(p=0.03)$. Relative to those with PDs and inducible WMAs, myocardial oxygen demand remained different in these individuals with PDs without an inducible WMA after accounting for LV afterload and contractility $(p=0.02$ and 0.03 respectively), but not after accounting for either LV stress related end diastolic volume index (LV preload) or resting concentricity $(p=0.31-0.71)$.

Conclusions: During dobutamine stress testing, elderly patients experience increased LV concentricity and declines in LV preload and myocardial oxygen demand, all of which are associated with an absence of inducible LV WMAs indicative of myocardial ischemia. These findings provide insight as to why dobutamine associated wall motion analyses exhibit reduced sensitivity for identifying inducible ischemia in elderly.

Trial registration: This study was registered with Clinicaltrials.gov (NCT00542503).

Keywords: Dobutamine, Elderly, Ischemia

\footnotetext{
* Correspondence: ghundley@wakehealth.edu

'Department of Internal medicine, Section on Cardiology, Wake Forest

School of Medicine, Winston Salem, North Carolina 27157, USA

'Department of Radiology, Wake Forest School of Medicine, Winston Salem,

North Carolina 27157, USA

Full list of author information is available at the end of the article
} 


\section{Background}

The presence of inducible left ventricular wall motion abnormalities (LVWMA) observed during dobutamine stress echocardiography (DSE) and dobutamine cardiovascular magnetic resonance (DCMR) is utilized widely to identify myocardial ischemia indicative of coronary artery disease (CAD) and forecast future cardiovascular (CV) events in those unable to exercise [1-5]. Dobutamine stress wall motion analyses performed with DSE or DCMR exhibit reduced sensitivity for detecting myocardial ischemia in those with altered left ventricular (LV) geometry due to increased LV concentricity and hypertrophy, both common conditions in the elderly [6,7]. Dobutamine stress myocardial perfusion analyses performed with contrast echocardiography or cardiovascular magnetic resonance (CMR) display improved sensitivity for identifying coronary artery stenosis of intermediate severity or forecasting prognosis regardless of ventricular shape [8-10].

In this study, we sought to understand the mechanism by which myocardial perfusion defects occur without concomitant inducible LVWMA in older individuals undergoing dobutamine stress testing. To accomplish this, we performed DCMR, a procedure which allows simultaneous assessment of wall motion and perfusion, LV concentricity, preload, afterload, and contractility in older individuals who frequently exhibit altered LV remodeling.

\section{Methods}

\section{Study design}

The study was approved by the Institutional Review Board of Wake Forest Health Sciences, and each participant provided witnessed written informed consent. This study was registered within the United States on Clinicaltrials.gov (NCT00542503) and was performed in accordance with the National Institutes of Health grants R01HL076438 and P30AG21332. In addition, this study complied with the Declaration of Helsinki. The purpose of this initiative was to utilize advanced DCMR techniques to identify rest and stress-induced cardiac and vascular abnormalities in older individuals that are associated with $\mathrm{CV}$ disease. In this study, each participant underwent an interview to collect and record information pertinent to $\mathrm{CV}$ disease (e.g., demographics, CV risk factors, etc.) and then underwent DCMR in which rate pressure product (RPP), aortic pulse wave velocity (PWV), LV myocardial oxygen demand (pressure volume area, PVA, stroke work, SW), mass, volumes, concentricity, and the presence of WMA and first pass gadolinium enhanced perfusion defects (PDs) indicative of ischemia were measured and recorded.

\section{Study population}

The study included participants from the rural counties of Central and Western North Carolina that exhibited a $>5$ years presence of risk factors (e.g., hypertension, diabetes, or CAD) for a cardiac event, but no history of myocardial infarction within 6 months of enrollment, no contraindication to intravenous dobutamine or gadolinium based contrast nor the performance of a CMR exam (such as the presence of incompatible bio-metallic implants or claustrophobia). None of the subjects had angina or symptoms of heart failure in the 12 months prior to enrollment. Recruitment of study participants was achieved through newspaper and television advertisements, or mailings to randomly selected individuals over the age of 55, but $<85$ years within Forsyth, Davie, and Davidson counties of Northwest North Carolina [4,5,11].

\section{Wall motion imaging}

The DCMR protocol was accomplished according to previously published techniques $[4,5]$ with images that were acquired on a 1.5-T (Siemens Avanto) whole-body imaging system using a phased-array cardiothoracic surface coil placed on the chest. Dobutamine was infused incrementally from low $(7.5 \mathrm{mcg} / \mathrm{kg} / \mathrm{min}$ ) to peak dose (20 to $40 \mathrm{mcg} / \mathrm{kg} / \mathrm{min}$ ), along with atropine (up to $1.5 \mathrm{mg}$ ), to achieve $80 \%$ of the maximum predicted heart rate response (MPHRR) for age. Cine images of the left ventricle were obtained in multiple contiguous short axis slices (apex to base) and in 3 long axis views (2, 3, and 4 chamber) at rest, low and peak dose dobutamine, and then after 10 minutes of recovery. Measurements of brachial artery systolic (SBP) and diastolic blood pressure (DBP) were performed using a CMR compatible sphygmomanometer.

According to previously published techniques [11,12], LV volumes were measured from the short-axis series of cine white blood imaging sequences using a modified Simpson's rule method [12]. LV concentricity was measured as the ratio of the LV mass to the LV end-diastolic volume as described by Chuang et al. [13]. Image acquisition parameters included a $45 \mathrm{msec}$ repetition time (TR), a $1 \mathrm{msec}$ echo time (TE), a $78^{\circ}$ flip angle (FA), a $400 \times 324 \mathrm{~mm}$ field of view (FOV), a $192 \times 109$ matrix, and an $8 \mathrm{~mm}$ thick slice with a $2 \mathrm{~mm}$ gap and an acceleration factor of 2 [5].

\section{Wall motion (WM) analysis}

The LV wall motion at rest, peak dose and in recovery was assessed with a visual scoring system in which $1=$ normal wall motion, 2 = hypokinesis, 3 = akinesis, and $4=$ dyskinesis. An LV inducible WMA was defined as an increase in score of $\geq 1$ (e.g., normal to hypokinetic) in 1 or more myocardial segments. Segments with an LV wall motion score of 2 or 3 at rest with no worsening of wall motion were considered negative for ischemia.

\section{Infarct imaging}

Ten minutes after the administration of gadobenate dimeglumine (Multihance) contrast, late gadolinium enhanced 
(LGE) inversion recovery images with steady state free precession readout were collected in the same short axis planes used to assess LV volumes. The sequence parameters included a $6 \mathrm{~mm}$ thick slice with a $2 \mathrm{~mm}$ gap, an $800 \mathrm{msec}$ TR, a $1.7 \mathrm{msec}$ TE, a $40^{\circ} \mathrm{FA}$, a $360 \times 270 \mathrm{~mm}$ FOV, and a $192 \times 109$ matrix with an inversion time adjusted to null the myocardium. Enhanced ( $>3$ standard deviations in mean signal intensity above background nonenhanced) regions were identified.

\section{Perfusion imaging}

First pass perfusion imaging with gadobenate dimeglumine $(0.1 \mathrm{mmol} / \mathrm{kg})$ was performed when $80 \%$ MPHRR was achieved. Eighty percent (80\%) of the maximum predicted heart rate response for age was selected for this study because [5] this heart rate response was previously shown to be accurate for identifying ischemia and forecasting cardiac prognosis [5,14]. A potential benefit of the mild decrease in peak stress heart rate $(80 \%$ relative to $85 \%$ ), is that it allowed for acquisition of 2 slices for assessing myocardial first pass perfusion at peak stress. These perfusion images were collected in the short axis orientation in the middle and apical segments ( 2 slice positions due to the rapid HR). Image parameters included an $8 \mathrm{~mm}$ thick slice, a $169 \mathrm{msec}$ TR, a $1.1 \mathrm{msec}$ TE, a FA of $12^{\circ}$, a FOV of $360 \times 270 \mathrm{~mm}$ and a $192 \times 108$ matrix. Rest first-pass perfusion imaging was not performed.

For each LV myocardial segment, a two-step process was utilized to identify perfusion defects indicative of ischemia. First, regions of first-pass hypo-perfusion were measured as a percentage of the corresponding myocardial wall thickness for that LV myocardial segment in the same imaging plane (Figure 1). The radial length of the PD was expressed as a percentage of the total LV myocardial wall thickness. In addition, the duration (or number of frames) for each PD was calculated from onset of LV myocardial enhancement until complete resolution of the defect. Any PD that persisted for more than 5 frames from onset of myocardial enhancement and encompassed $>25 \%$ of the thickness of the wall was further evaluated for classification as ischemic [15]. This criterion of $25 \%$ transmural involvement was used successfully by other investigators to exclude dark rim artifacts $[16,17]$.

Further evaluation was performed to exclude PDs related to prior infarcted territories. The extent of these PDs suspected of ischemia was compared to the extent of infarction as assessed in the LGE images within the same myocardial segments. Participants with PDs that extended beyond the areas of LGE or occurred in a territory or segment that differed from the segmental territory in which the LGE was noted were classified as ischemic. Any PD that matched the area of LGE was not classified as ischemic.

\section{Aortic stiffness assessment using pulse wave velocity}

According to previously published techniques [18], PWV was assessed using phase-contrast cardiovascular magnetic resonance (PC-CMR). Images of the proximal thoracic aorta were obtained in an axial cross-sectional plane placed at the apex of the main pulmonary artery (identified with a sagittal localizer). PC-CMR imaging parameters included an $8 \mathrm{~mm}$ thick slice, a $10 \mathrm{msec}$ TR, a 3-5 msec TE, a $15-20^{\circ} \mathrm{FA}, 20 \mathrm{~ms}$ temporal resolution, a 340 to $360 \mathrm{~mm}$ FOV, a $256 \times 192$ matrix and a through plane velocity encoding of $150 \mathrm{~cm} / \mathrm{sec}$. Pulse wave velocity (PWV) was calculated by dividing the distance between the ascending and descending thoracic aorta by the transit time of the flow wave [18].

\section{Measures of global myocardial oxygen demand}

In addition to the changes in HR, blood pressure (BP) and the RPP, we also assessed the LV stroke work (SW) and the systolic PVA, a measure indicating the global LV myocardial oxygen demand $[19,20]$. The systolic PVA is defined as both the mechanical LV SW and the mechanical

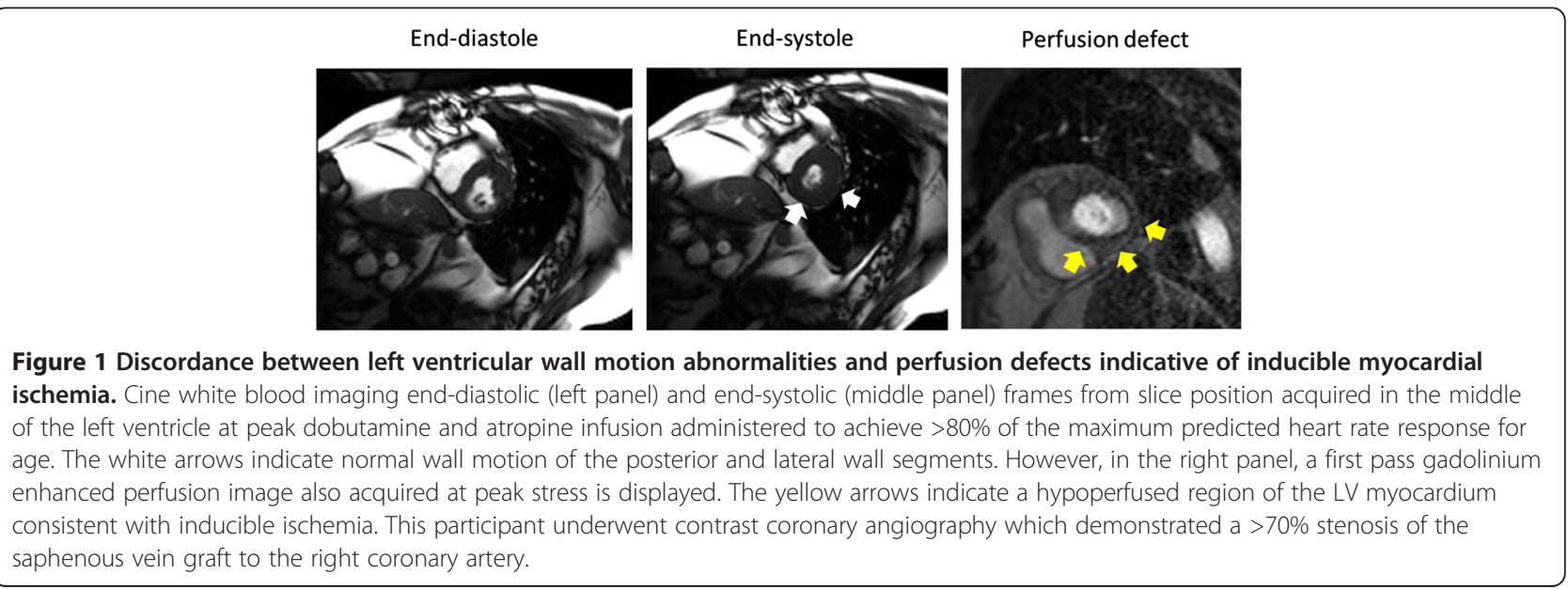


potential energy which is expended during systole [21,22]. This is the area enclosed by the slopes of the end-systolic and the end-diastolic pressure volume relationship [22]. Invasive studies have shown an excellent correlation between the PVA and the myocardial oxygen consumption under different loading conditions and with dobutamine. The SW was defined as the mean arterial pressure * LV stroke volume. The systolic PVA was defined as: LV $\mathrm{SW}+1 / 2^{*} \mathrm{LV}$ end-systolic pressure * end-systolic volume. $\mathrm{LV}$ end systolic pressure was calculated as $0.85^{*}$ brachial systolic blood pressure (SBP) [23].

\section{Statistical analysis}

In accordance with the American Heart Association Scientific Statement [24], the 10 middle and apical LV myocardial segments were assessed for LV first pass PDs, LGE, and inducible WMA. Participants in this study were characterized into one of 3 groups including those: 1) without a PD or WMA (Group I), 2) with a PD but without a WMA (Group II), and 3) with both a PD and a WMA indicative of ischemia (Group III). The differences in demographic, hemodynamic, CMR volumetric parameters, and indices of myocardial oxygen demand between the 3 Groups were assessed by an analysis of variance test of equality (ANOVA). In Tables 1 and 2, the p-values are displayed for the overall equality of the three groups. In the Figures, the comparisons between specific groups were accomplished using pairwise comparisons. A p-value of $<0.05$ was considered significant for either forms of testing. The differences in the LV myocardial SW and the PVA were adjusted for preload (resting and peak dose LV end-diastolic volume index or LVEDVi), afterload (peak dose PWV), contractility (LV ejection fraction or LVEF), and LV concentricity using analysis of covariance. Multiple regression models adjusting for SBP, LVEDV baseline and LVEDV peak dose were selected and reported by stepwise regression. The sensitivity and specificity of dobutamine related wall motion abnormalities for detecting obstructive $C A D$ was assessed with the results of the perfusion component of the DCMR protocol serving as the reference standard. Results were expressed as means \pm standard error of the estimate unless stated otherwise.

\section{Results}

Two hundred seventy-eight (278) consecutive participants were enrolled into the study; the demographic data from the participants are shown in Table 1. No differences in age, gender, BMI, prevalence of hypertension, diabetes, CAD, dyslipidemia, smoking, or medication use were noted between the 3 Groups ( $p=0.05$ to 0.54 for all; Table 1). Analyzing the data using pairwise comparisons, there were differences between individuals in Groups II and III versus those in Group I regarding the incidence of prior revascularization $(32 \%$ and $44 \%$ vs.
$13 \%$ respectively, $\mathrm{p}<0.001$ for both comparisons); and prior MI ( $17 \%$ vs. $18 \%$ vs. $7 \%$ respectively; $\mathrm{p}=0.05$ for both comparisons). There were no differences between Groups II and III regarding the incidence or prior MI or revascularization ( $\mathrm{p}=0.98$ and $\mathrm{p}=0.29$ respectively).

According to previously published criteria, $64 \%$ of the participants exhibited LV concentricity and $82 \%$ had left ventricular hypertrophy. Consistent with previously published data [25], in the population aged 70 and older, women compared to men had a greater incidence of concentric ventricles $(80 \%$ vs. $42 \%, \mathrm{p}<0.001$ while in those aged less than 70 there were no gender differences in the incidence of concentric ventricles $(61 \%$ vs. $61 \%$, $\mathrm{p}=0.87$ ). However women had a higher incidence of LVH compared to men ( $96 \%$ vs. $62 \%$ p $<0.001)$.

Forty-six participants exhibited a PD indicative of ischemia. Of the 46 subjects with PDs, 18 had corresponding inducible LV WMAs (Group III) [13]. The remaining 28 exhibited a PD but no WMA (Group II). No participants experienced an inducible LVWMA without a CMR perfusion defect. The presence of a DCMR inducible LVWMA exhibited a sensitivity of $39 \%$ and a specificity of $100 \%$ for the detection of a PD consistent with inducible LV myocardial ischemia. Example cases from participants with concordance and discordance between WMA and PDs are shown in Figures 1 and 2.

Of the 28 patients with perfusion defects consistent with ischemia in Group II, 6 had peri-infarct ischemia (i.e. a PD extending beyond proportion to the region of LGE), 3 had LGE in a different territory and 19 had no LGE. Of the 18 patients in Group III, 9 had periinfarct ischemia, 2 had LGE in a different territory and 7 had no LGE. Compared to the subjects in Group II, those in Group III trended toward larger trans-mural extents of their PDs $(29 \%$ vs. $33 \%, p=0.26)$, somewhat longer duration PDs (10 frames vs. 13 frames, $\mathrm{p}=0.11$ ), and more segments with LGE (11 vs. 18, p = 0.04). Those in Group III did exhibit a greater number of WMAs indicative of ischemia ( 1 vs. $4, \mathrm{p}<0.001$ segments, respectively).

The cardiac chamber volumes and function, arterial stiffness measures, and hemodynamic parameters of the participants at rest and with stress are shown in Table 2. Systolic blood pressure (SBP), diastolic blood pressure (DBP), pulse pressure (PP), HR and the rate pressure product (RPP) were similar at rest and with stress among the 3 Groups $(p=0.22$ to 0.77$)$. Overall, with intravenous dobutamine, the HR rose $96 \%$, the SBP decreased $6 \%$ and the RPP rose $85 \%$ for the participants in the study with no difference between the three groups $(\mathrm{p}=0.63,0.74$, and 0.92 respectively).

At rest, those in Group I exhibited a higher LVSV index (LVSVi) and a higher cardiac index compared to those in Groups II and III ( $\mathrm{p}=0.03$ for both indices). No differences between the 3 groups were noted in the 
Table 1 Demographic data

\begin{tabular}{|c|c|c|c|c|}
\hline Category & $\begin{array}{l}\text { Group I No LV perfusion } \\
\text { defect or wall motion } \\
\text { abnormality }(n=232)\end{array}$ & $\begin{array}{l}\text { Group II LV perfusion defect } \\
\text { present, no wall motion } \\
\text { abnormality }(n=28)\end{array}$ & $\begin{array}{l}\text { Group III LV perfusion } \\
\text { defect and wall motion } \\
\text { abnormality present }(n=18)\end{array}$ & p-value* \\
\hline Age (years) & $68 \pm 8$ & $70 \pm 9$ & $69 \pm 8$ & 0.32 \\
\hline Men (\%) & $110(47 \%)$ & $14(50 \%)$ & $14(78 \%)$ & 0.046 \\
\hline Race/Ethnicity & & & & 0.50 \\
\hline Caucasian & $171(74 \%)$ & $23(82 \%)$ & $16(89 \%)$ & \\
\hline African Am. & $56(24 \%)$ & $4(14 \%)$ & $2(11 \%)$ & \\
\hline Hispanic & $2(1 \%)$ & $1(4 \%)$ & $0(0 \%)$ & \\
\hline Asian & $3(1 \%)$ & $0(0 \%)$ & $0(0 \%)$ & \\
\hline Body mass index (m/kg2) & $30.4(6.5)$ & $30.3(4.8)$ & $32.1(5.4)$ & 0.54 \\
\hline Hypertension & $203(88 \%)$ & $27(96 \%)$ & $16(89 \%)$ & 0.38 \\
\hline Coronary artery disease & $57(25 \%)$ & $9(32 \%)$ & $8(44 \%)$ & 0.14 \\
\hline Prior Ml & $16(7 \%)$ & $5(17 \%)$ & $3(18 \%)$ & 0.05 \\
\hline Prior revascularization ( $\mathrm{PCl}$ or $\mathrm{CABG}$ ) & $31(13 \%)$ & $9(32 \%)$ & $8(44 \%)$ & $<0.001$ \\
\hline Diabetes & $94(41 \%)$ & $11(39 \%)$ & $8(44 \%)$ & 0.94 \\
\hline Hypercholesterolemia & $152(70 \%)$ & $22(79 \%)$ & $12(86 \%)$ & 0.30 \\
\hline Smoking & $90(42 \%)$ & $10(36 \%)$ & $6(43 \%)$ & 0.79 \\
\hline \multicolumn{5}{|l|}{ Medications } \\
\hline Angiotensin converting enzyme inhibitor & $99(43 \%)$ & $15(54 \%)$ & $7(39 \%)$ & 0.52 \\
\hline Angiotensin receptor blocker & $64(30 \%)$ & $5(18 \%)$ & $3(20 \%)$ & 0.30 \\
\hline Statin & $152(66 \%)$ & $21(75 \%)$ & $14(78 \%)$ & 0.37 \\
\hline Beta blocker & $99(43 \%)$ & $13(46 \%)$ & $11(61 \%)$ & 0.32 \\
\hline Calcium channel antagonist & $68(30 \%)$ & $8(29 \%)$ & $9(50 \%)$ & 0.20 \\
\hline
\end{tabular}

*Analysis of variance test of equality of three groups. Summary statistics are mean (standard error) for continuous variables and frequency (\%) for categorical variables. Abbreviations: CABG, coronary artery bypass grafting; LV, left ventricular; $\mathrm{MI}$, myocardial infarction; $\mathrm{PCl}$, percutaneous coronary intervention.

LV end-systolic volume index (LVESVi) or the LVEF at rest. Those in Group III possessed the highest concentricity index $(\mathrm{p}=0.02)$ compared to those in Groups I and II with no differences in the LV mass index between the three groups $(p=0.35)$. With dobutamine, all three groups experienced a similar decrease in the LVEDVi. As expected, individuals in Group III exhibited the lowest stress LVEF and the highest stress LVESVi values relative to those in Groups I and II ( $\mathrm{p}=0.002$ and $\mathrm{p}=0.001$ respectively). PWV was not different among the groups at rest $(\mathrm{p}=0.76)$ or with stress. $(\mathrm{p}=0.14)$.

The indices of myocardial oxygen demand at rest are shown in Figure 3 and at stress in Figure 4. At rest as shown in Figure 3, the left ventricular pressure volume area (PVA) and stroke work (SW) of individuals in Group II were lower than those in Group I $(9,016 \pm 559$ vs. $10,439 \pm 201 \mathrm{mmHg}^{*} \mathrm{ml}, \mathrm{p}=0.02$ and $6,594 \pm 417$ vs. $7,794 \pm 150 \mathrm{mmHg} * \mathrm{ml}, \mathrm{p}=0.007$ respectively) and lower than those in Group III $(9016 \pm 559$ vs. $10,618 \pm$ $768 \mathrm{mmHg} * \mathrm{ml}, \mathrm{p}=0.09$ and $6,594 \pm 417$ vs. $7708 \pm$ $569 \mathrm{mmHg}$ *ml, $\mathrm{p}=0.01$ respectively). However there were no differences in PVA and SW between Groups I and II with dobutamine $(7,660 \pm 645$ vs. $8,409 \pm 227 \mathrm{mmHg}$ "ml, $\mathrm{p}=0.27$ and $6,160 \pm 524$ vs. $6,877 \pm 187 \mathrm{mmHg*ml}$, $\mathrm{p}=0.25$ respectively). In contrast, during stress, the PVA and SW in Group II were lower when compared to Group III $\left(7660 \pm 645\right.$ vs. $10,023 \pm 862 \mathrm{mmHg}^{*} \mathrm{ml}, \mathrm{p}=0.03$, and $6,160 \pm 524$ vs. $7682 \pm 715 \mathrm{mmHg} * \mathrm{ml}, \mathrm{p}=0.09$ ), respectively.

To determine the factors influencing this lower myocardial oxygen demand between groups II and III, we adjusted the left ventricular PVA and SW for differences in baseline and peak stress LVEDVi (preload), peak stress PWV (afterload), the resting LVEF (contractility), and the LV concentricity as shown in Figure $4 \mathrm{~A}$ and B respectively. As shown in Figure 4A, the lower LVPVA in individuals in Group II relative to Group III persisted after adjusting for contractility $(7,684 \pm 649$ vs. $10,109 \pm$ $\left.883 \mathrm{mmHg}^{*} \mathrm{ml}, \mathrm{p}=0.03\right)$, and afterload $(7,639 \pm 682$ vs. $10,421 \pm 930 \mathrm{mmHg} * \mathrm{ml}, \mathrm{p}=0.02)$. However, the differences in the PVA after dobutamine between individuals in Groups II and III were eliminated after accounting for stress LVEDVi $(p=0.71$, Figure $4 A)$, and LV concentricity $(p=0.31$; Figure $4 A)$. Similarly, the lower LVSW in individuals in Group II relative to Group III persisted after adjusting for contractility $(6,206 \pm 529$ vs. $8123 \pm$ $720 \mathrm{mmHg}{ }^{*} \mathrm{ml}, \mathrm{p}=0.03$; Figure $4 \mathrm{~B}$ ), and nearly persisted 
Table 2 Hemodynamics and CMR indices

\begin{tabular}{|c|c|c|c|c|}
\hline Measure & Group I $(n=232)$ & Group II (n= 28) & Group III $(n=18)$ & p-value ${ }^{*}$ \\
\hline \multicolumn{5}{|l|}{ Rest } \\
\hline Heart rate (beats/min.) & $65(0.74)^{* *}$ & $63.64(2.06)$ & $65.73(2.81)$ & 0.77 \\
\hline Systolic blood pressure (mmHg) & $139.81(1.18)$ & $137.29(3.29)$ & $136.67(4.58)$ & 0.64 \\
\hline Diastolic blood pressure (mmHg) & $79.53(0.82)$ & $75.29(2.29)$ & $78.80(3.13)$ & 0.22 \\
\hline Mean arterial pressure $(\mathrm{mmHg})$ & $99.58(0.82)$ & $95.95(2.27)$ & $98.09(3.21)$ & 0.30 \\
\hline Rate pressure product & $9108(133)$ & $8753(369)$ & $8962(504)$ & 0.65 \\
\hline Left ventricular end diastolic volume index $\left(\mathrm{ml} / \mathrm{m}^{2}\right)$ & $62.2(1.01)$ & $56.7(2.81)$ & $59.3(3.91)$ & 0.17 \\
\hline Left ventricular end systolic volume index $\left(\mathrm{ml} / \mathrm{m}^{2}\right)$ & $22.2(0.52)$ & $21.3(1.31)$ & $22.8(1.72)$ & 0.72 \\
\hline Left ventricular stroke volume index $\left(\mathrm{ml} / \mathrm{m}^{2}\right)$ & $39.4(0.61)$ & $35.5(1.52)$ & $36.5(2.11)$ & 0.03 \\
\hline Ejection fraction (\%) & $64.2(0.51)$ & $62.7(1.41)$ & $62.5(1.82)$ & 0.46 \\
\hline Cardiac index $\left(\mathrm{ml} / \mathrm{min} / \mathrm{m}^{2}\right)$ & $2536(39)$ & $2239(109)$ & $2380(149)$ & 0.03 \\
\hline Pulse wave velocity (m/s) & $11(0.47)$ & $9.75(1.28)$ & $9.96(1.75)$ & 0.76 \\
\hline Left ventricular mass index $\left(\mathrm{gm} / \mathrm{m}^{2}\right)$ & $66(14)$ & $65.9(11.32)$ & $71.1(12.41)$ & 0.35 \\
\hline Left ventricular concentricity & $1.09(0.02)$ & $1.19(0.05)$ & $1.26(0.07)$ & 0.02 \\
\hline \multicolumn{5}{|l|}{ Stress } \\
\hline Peak heart rate (beats/min.) & $127(1.12)$ & $129.81(3.16)$ & $126.92(4.39)$ & 0.63 \\
\hline Peak systolic blood pressure $(\mathrm{mmHg})$ & $130(1.75)$ & $126.37(4.92)$ & $131.93(6.84)$ & 0.74 \\
\hline Peak diastolic blood pressure $(\mathrm{mmHg})$ & $70.79(1.21)$ & $68.04(3.40)$ & $75.14(4.72)$ & 0.47 \\
\hline Peak mean arterial pressure $(\mathrm{mmHg})$ & $90.57(1.27)$ & $87.48(3.58)$ & $94.07(4.97)$ & 0.54 \\
\hline Peak rate pressure product & $16400(251)$ & $16381(706)$ & $16804(1982)$ & 0.92 \\
\hline Left ventricular end diastolic volume index $\left(\mathrm{ml} / \mathrm{m}^{2}\right)$ & $51.60(0.78)$ & $50.99(2.15)$ & $54.97(2.98)$ & 0.52 \\
\hline Left ventricular end systolic volume index $\left(\mathrm{ml} / \mathrm{m}^{2}\right)$ & $13.68(0.33)$ & $14.53(0.87)$ & $18.36(1.24)$ & 0.001 \\
\hline Left ventricular stroke volume index (SVI) $\left(\mathrm{ml} / \mathrm{m}^{2}\right)$ & $37.85(0.62)$ & $36.23(1.69)$ & $36.64(2.35)$ & 0.61 \\
\hline Ejection fraction (\%) & $73.3(0.50)$ & $71.2(1.42)$ & $66.5(1.93)$ & 0.002 \\
\hline Cardiac index $\left(1 / \mathrm{min} / \mathrm{m}^{2}\right)$ & $4800(83)$ & $4688(232)$ & $4644(316)$ & 0.82 \\
\hline Pulse wave velocity $(\mathrm{m} / \mathrm{s})$ & $11.51(0.61)$ & $12.44(1.69)$ & $16.27(2.39)$ & 0.14 \\
\hline
\end{tabular}

*Analysis of variance test of equality of three groups. The p-values presented are an overall test that the three groups are equal. Abbreviations:, EDV, End diastolic volume; EF, Ejection fraction; PVA, Pressure volume area; PWV, Pulse wave velocity; SW, Stroke work.
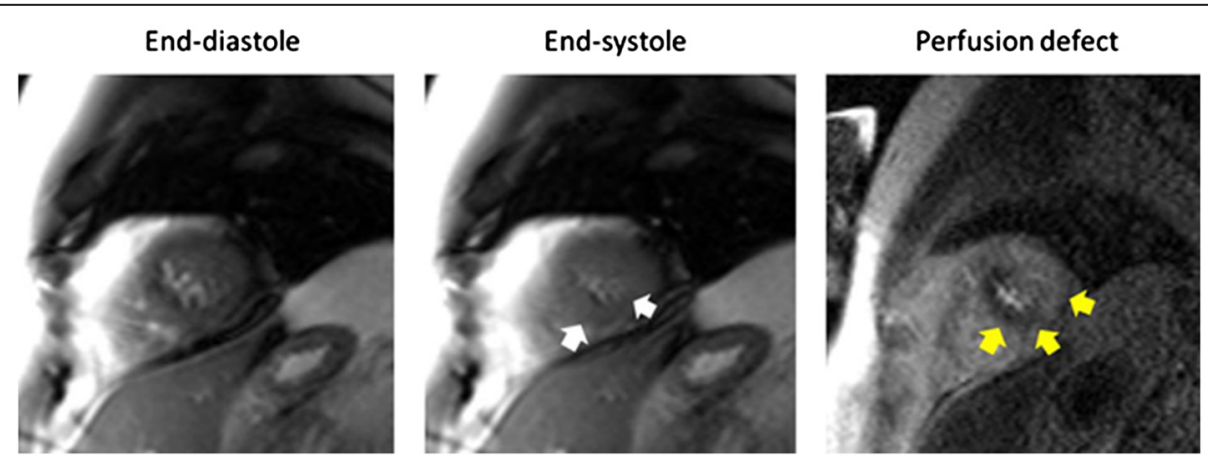

Figure 2 Concordance between left ventricular wall motion abnormalities and perfusion defects indicative of inducible myocardial ischemia. Cine white blood imaging end-diastolic (left panel) and end-systolic (middle panel) frames from slice position acquired in the apex of the left ventricle at peak dobutamine and atropine infusion administered to achieve $>80 \%$ of the maximum predicted heart rate response for age. The white arrows indicate a wall motion abnormality in the inferoseptum as manifest by incomplete LV cavity obliteration at peak stress. In the right panel, a first pass gadolinium enhanced perfusion image also acquired at peak stress is displayed. The yellow arrows indicate a hypoperfused region of the LV myocardium consistent with inducible ischemia. In this case there was concordance of the wall motion and perfusion analyses both indicating inducible ischemia. 


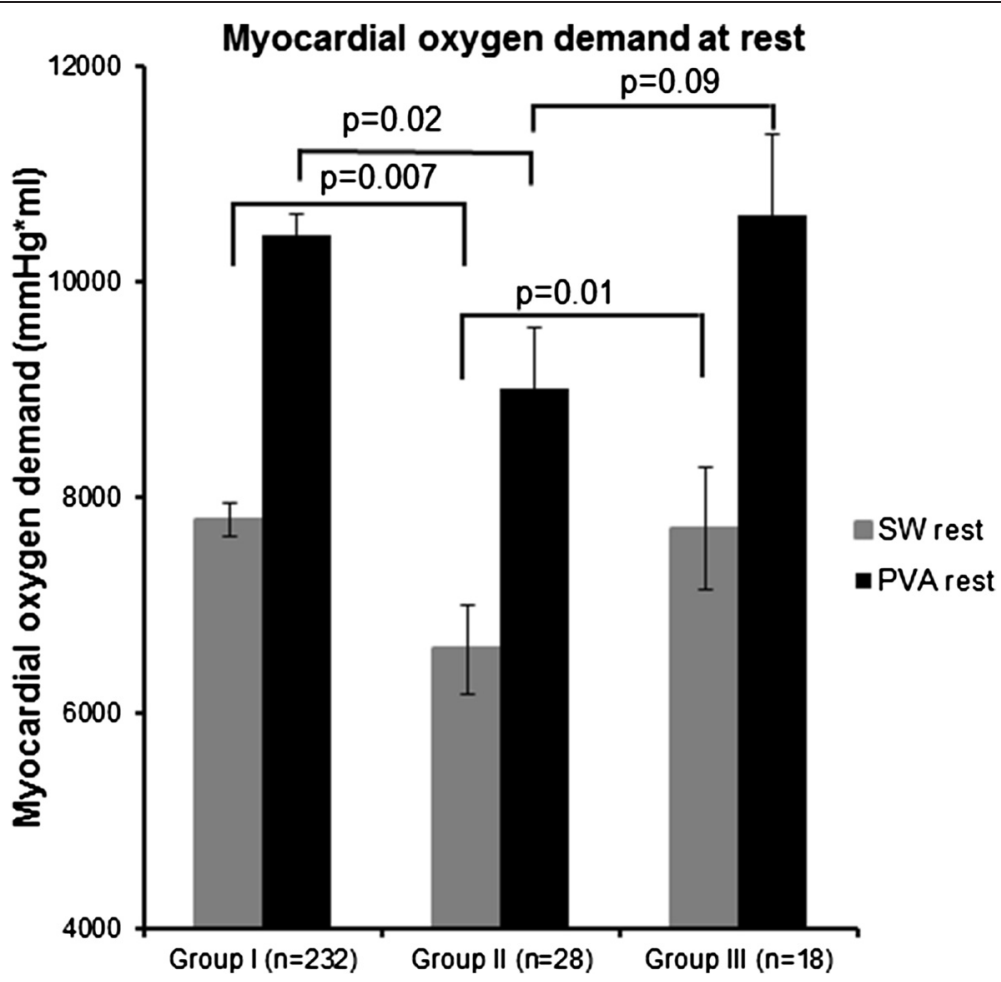

Figure 3 Resting measures of myocardial oxygen demand between the three groups. The mean \pm the standard error of the myocardial oxygen demand (Y-axis) and the number of participants in each of the 3 study groups (X-axis) are shown. The myocardial stroke work is denoted by the gray bars, and the pressure volume area is denoted by the black bars. At rest, Group II participants (wall motion negative, perfusion positive) have lower myocardial oxygen demand as assessed by myocardial stroke work (SW) than those in III (wall motion and perfusion positive) or Group I. Also, PVA is reduced in Groups II relative to Group I and trends toward a difference with Group III.

after accounting for LV afterload (6,170 \pm 556 vs. 7,982 \pm $776 \mathrm{mmHg}$ *ml, $\mathrm{p}=0.06$; Figure $4 \mathrm{~B}$ ), but as shown in Figure $4 \mathrm{~B}$, were eliminated when adjusted for stress preload, LVEDVi ( $\mathrm{p}=0.34$ ) and LV concentricity $(\mathrm{p}=0.47)$. This suggests that the lower LVSW and the PVA seen in individuals in Group II are related to the LV preload and LV concentricity. Similar results between Groups II and III were noted when the PVA and SW were respectively adjusted for baseline LVEDVI $(8,207 \pm 555$ vs. 9,534 \pm $740 \mathrm{mmHg} * \mathrm{ml}, \mathrm{p}=0.15$, and $6,586 \pm 459$ vs. $7,294 \pm$ $624 \mathrm{mmHg}^{*} \mathrm{ml}, \mathrm{p}=0.36$.

\section{Discussion and conclusions}

There are three important findings related to this study. First, in older men and women receiving a pharmacologic dobutamine stress test, myocardial oxygen demand often decreases during receipt of intravenous dobutamine despite achieving $80 \%$ of the MPHRR for age (Figure 4A). Second, this reduction in myocardial oxygen demand is related to altered LV geometry and reductions in LV preload that are manifested by a decrease in LVEDV upon receipt of intravenous dobutamine (Figure 4A and B). Third, dobutamine related reductions in myocardial oxygen demand observed in older men and women may confound the identification of LV inducible wall motion abnormalities indicative of ischemia (Figures 1, 2, 3 and 4). In this study of elderly subjects at risk for inducible ischemia, of those exhibiting PDs suggestive of ischemia, $60 \%$ of individuals did not demonstrate a corresponding inducible LV wall motion abnormality.

As shown in Figures 1 and 2 and Table 1, we identified three groups of individuals within the study population: those without (Group I) or with (Group III) inducible PDs and WMAs, and those with an inducible PD but without an inducible LV WMA indicative of ischemia (Group II). Similar observations in which PDs occur in the absence of dobutamine induced WMA have been reported in patients with resting LV hypertrophy [26]. In those with LV hypertrophy, the presence of PDs has an increased sensitivity when compared to inducible LV WMA for detection of coronary arterial luminal narrowings of $>70 \%$ [26]. This is similar to observations made in studies of patients with altered LV geometry using both echocardiography and CMR [6,7]. The addition of myocardial perfusion has previously exhibited diagnostic and prognostic value with dobutamine stress testing, regardless of whether echocardiography $[7,8]$ or CMR imaging $[27,28]$ was used for diagnosis. 
A Myocardial oxygen demand at stress: PVA

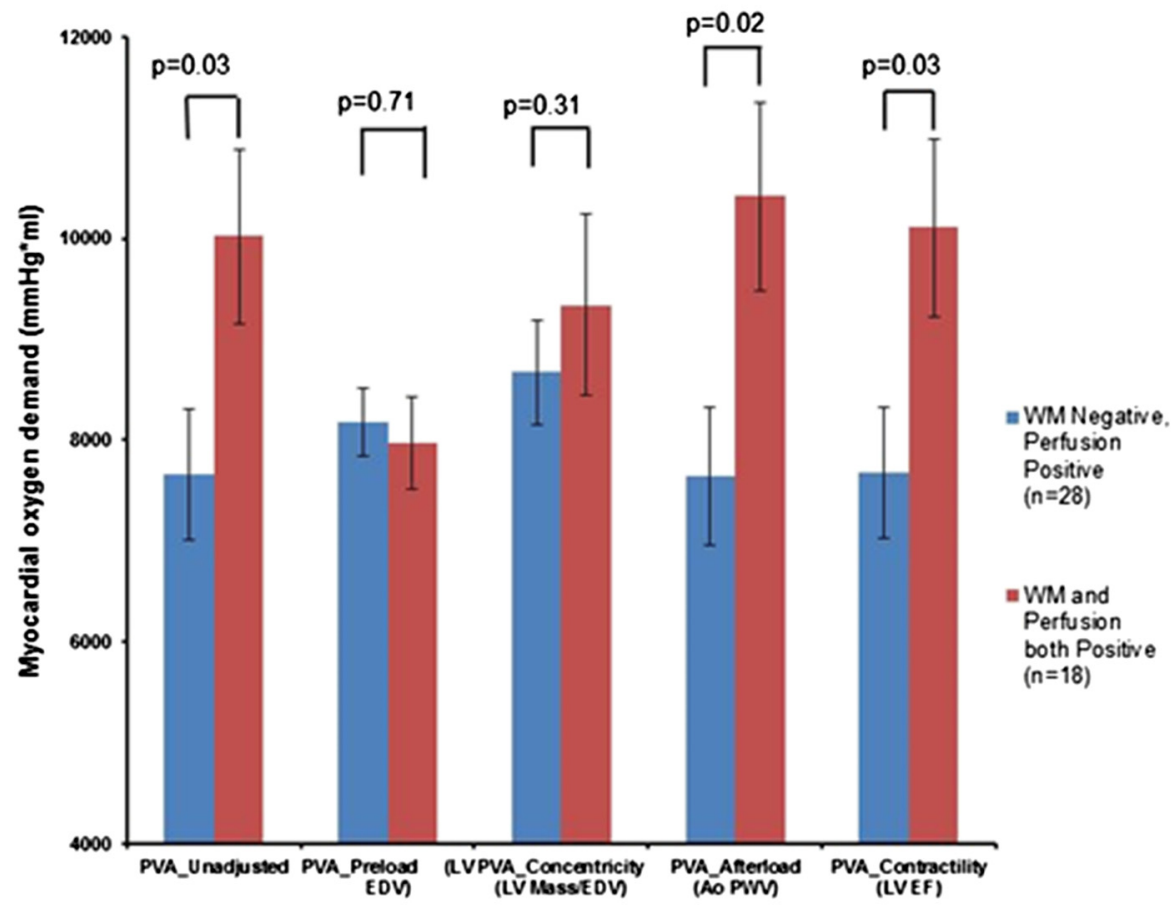

B Myocardial oxygen demand at stress: SW

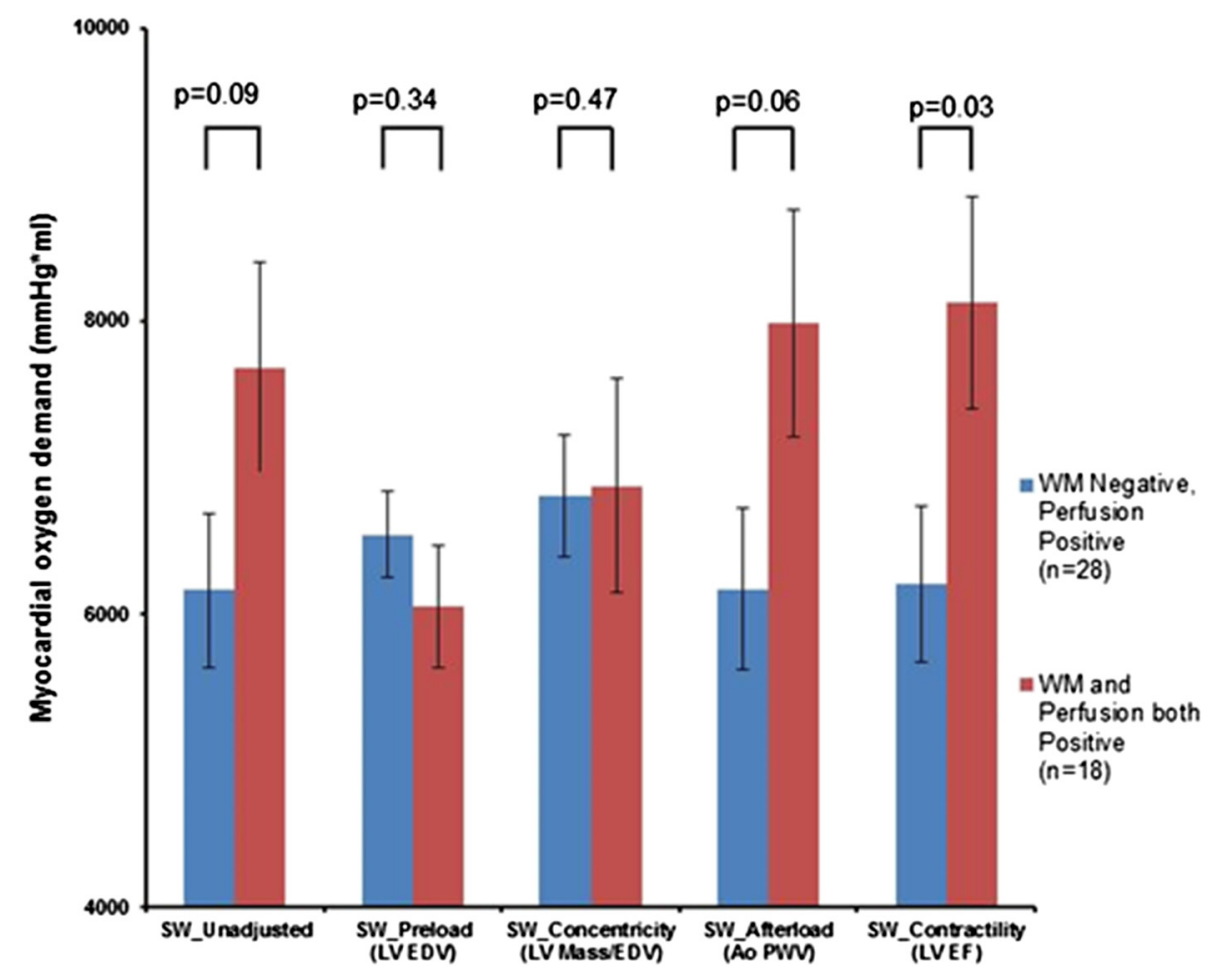

Figure 4 (See legend on next page.) 
(See figure on previous page.)

Figure 4 Adjusted and unadjusted measures of myocardial oxygen demand. A shows the unadjusted differences in myocardial oxygen demand with stress, specifically the PVA, between Groups II and II. The differences in the unadjusted PVA between Groups II and III are attenuated when adjusted for LV preload (LV End-diastolic volume (LVEDV)) and LV concentricity, but persist when adjusted for LV afterload (Aortic pulse wave velocity (PW) and LV contractility (LV ejection fraction (LVEF). B demonstrates the differences in Stroke work between Groups II and III. Similar to PVA, LV Preload and Concentricity determine the differences in the Stroke Work between Groups II and III, but not the LV afterload or contractility. A and $\mathbf{B}$ show the attenuation of these differences when adjusted for LV preload and concentricity respectively. This suggests that LV preload and concentricity are the main factors influencing myocardial oxygen demand.

To further understand why PDs occurred in the absence of WMA, we examined multiple hemodynamic variables throughout the stress testing procedure. As shown in Table 2, HR increased while SBP decreased in all 3 participant groups (Table 2). This decrease in SBP contrasts with observations in prior studies of younger individuals in which a hypertensive response was uniformly noted after receipt of dobutamine [29]. As a result, the RPPs of the participants in this study ranged from 16,000 to 17,000 which are somewhat lower values than reported in previous studies of dobutamine stress testing, even after accounting for participant age [26,30]. This observation may be related to the fact that elderly individuals often exhibit increased ventricular and arterial stiffness. As a result, their LV end-systolic pressure volume relationship curve is steeper which can potentially lead to greater reductions in SBP after the administration of systemic vasodilators when compared to younger individuals with more compliant arterial systems [30].

PWV, a measure of arterial stiffening, was mildly elevated at rest across the 3 participant Groups. With stress, PWV remained similar or increased in all 3 Groups with the largest increase occurring in Group III participants. These data indicate that overall, aortic stiffness was elevated in older subjects and remained elevated after receipt of dobutamine. Given the absence of a concomitant increase in SBP in Group III, one possible explanation of the high PWV is that this group did not experience a vasodilator response with dobutamine stress. We have shown that elderly subjects with diabetes and impaired fasting glucose do not experience a normal decrease in afterload with dobutamine and instead experience an increase in arterial stiffness as evidenced by a decrease in aortic distensibility [31].

As shown in Figure 4A, myocardial oxygen demand as assessed by PVA was diminished at peak stress in those individuals with PDs but no WMAs (Group II) relative to those individuals in Group III $(\mathrm{p}=0.03)$. We sought to determine which aspect (LV preload, LV afterload, LV contractility, or LV concentricity) of myocardial oxygen demand accounted for these differences by adjusting for each variable in our linear regression models. As shown in Figures $4 \mathrm{~A}$ and $\mathrm{B}$, after accounting LV preload and concentricity, the differences in myocardial oxygen demand between Groups II and II were eliminated. This implies that both of these variables were integral for establishing the difference in myocardial oxygen demand noted between these two Groups.

Since LV wall motion assessments of ischemia are driven by a supply-demand mismatch, a lower oxygen demand might not be adequate to provoke a WMA and could render the study insensitive for the detection of ischemia. The observation of a lower myocardial oxygen demand in those with increased LV concentricity, leading to hyperdynamic (and, consequently, and absence of WMA even though ischemia may be present during intravenous dobutamine) has been demonstrated by Mirelis, et al. [32] These investigators identified fewer DCMR WMA in individuals with increased LV concentricity.

Consistent with findings from other larger population based studies such as the Multi-Ethnic Study of Atherosclerosis, the elderly individuals in our group exhibited a smaller LVEDV and increased LV concentricity [28]. In addition, as reported in other studies, the LVEDVi decreased during dobutamine [32]. However, the magnitude of the decrease in LVEDVi was higher in this study relative to observations in prior studies of middle aged individuals undergoing dobutamine stress [11]. In the presence of concentric LV remodeling or hypertrophy, decreases in LV preload can reduce the LV cavity size and render it more difficult to visualize WMAs even when myocardial shortening is abnormal [29].

The results of this study have important implications for the interpretation of dobutamine wall motion stress testing in elderly individuals. As shown in this study, up to $61 \%$ of subjects who had ischemia identified by PDs did not experience an inducible WMA. In addition, the presence of these PDs was not associated with failure to achieve target HR during testing, or differences in the SBP response (which parenthetically decreased during testing) among all individuals receiving testing. Therefore, inducible ischemia (as defined by an inducible PD) may in fact be present during dobutamine stress in the elderly even though LV WMAs are absent. Other studies have found similar results regarding the lower sensitivity of LVWMA measures in assessing the risks for CAD [6,28].

Our study does exhibit some limitations. First, we did not perform contrast coronary angiography on the participants. Recently, however, the diagnostic utility and accuracy of CMR perfusion abnormalities have been 
established in multicenter and multivendor trials $[33,34,35]$. Second, the majority of our subjects were Caucasian. Insufficient numbers of individuals from other ethnicities were present to examine the effects of race or ethnicity. Third, our study population exhibited pre-existing CAD and multiple risk factors for CAD with either no or stable patterns of angina. More research is required to determine results in subjects with new onset, or unstable patterns of angina.

In conclusion, despite achieving target HR, a subset of elderly patients with risk factors for CAD who undergo dobutamine stress experience perfusion abnormalities indicative of ischemia without a concomitant LV wall motion abnormality. Mechanistically, this occurs in part due to a lower myocardial oxygen demand that appears related to reductions in $\mathrm{LV}$ preload and increases in LV concentricity.

\section{Competing interests}

The authors declare that they have no competing interest.

\section{Authors' contributions}

SV Analyzed the data and drafted the manuscript. WCL Provided critical input on data analysis and reviewed the manuscript. TM Performed statistical analysis of the data used in the manuscript and reviewed the manuscript. $\mathrm{RBS}, \mathrm{CH}, \mathrm{VT}$, CC Reviewed the manuscript. WGH Principal investigator of the study, analyzed the data, drafted the manuscript. All authors read and approved the final manuscript.

\section{Acknowledgments}

Contrast agent gadobenate dimeglumine (Multihance) was provided by Bracco Diagnostics.

\section{Funding}

This work was supported by the National Institutes of Health grants R01 HL076438, P30 AG21332, N01 HC95165, R01 CA167821, and R33 CA121296.

\section{Author details}

${ }^{1}$ Department of Internal medicine, Section on Cardiology, Wake Forest School of Medicine, Winston Salem, North Carolina 27157, USA. ${ }^{2}$ Department of Internal Medicine, University of Mississippi, Jackson, Mississippi 39216, USA ${ }^{3}$ Department of Biostatistical sciences, Wake Forest School of Medicine, Winston Salem North Carolina 27157, USA. ${ }^{4}$ Mid Carolina Cardiology, Charlotte North Carolina 28204, USA. ${ }^{5}$ Department of Biomedical Engineering, Wake Forest School of Medicine, Winston Salem, North Carolina 27157, USA. 'Aurora Cardiovascular Services, Milwaukee, Wisconsin 53215, USA. ${ }^{7}$ Department of Radiology, Wake Forest School of Medicine, Winston Salem, North Carolina 27157, USA.

Received: 11 September 2014 Accepted: 13 March 2015 Published online: 08 April 2015

\section{References}

1. Sawada SG, Segar DS, Ryan T, Brown SE, Dohan AM, Williams R, et al Echocardiographic detection of coronary artery disease during dobutamine infusion. Circulation. 1991;83:1605-14.

2. Van Damme H, Piérard L, Gillain D, Benoit T, Rigo P, Limet R. Cardiac risk assessment before vascular surgery: A prospective study comparing clinica evaluation, dobutamine stress echocardiography, and dobutamine tc-99 m sestamibi tomoscintigraphy. Cardiovasc Surg. 1997;5:54-64.

3. Mazeika PK, Nadazdin A, Oakley CM. Dobutamine stress echocardiography for detection and assessment of coronary artery disease. J Am Coll Cardiol. 1992:19:1203-11.

4. Hamilton CA, Link KM, Salido TB, Epstein FH, Hundley WG. Is imaging at intermediate doses necessary during dobutamine stress magnetic resonance imaging? J Cardiovasc Magn Reson. 2001;3:297-302.
5. Hundley WG, Morgan TM, Neagle CM, Hamilton CA, Rerkpattanapipat $P$, Link KM. Magnetic resonance imaging determination of cardiac prognosis. Circulation. 2002;106:2328-33.

6. Gebker R, Mirelis JG, Jahnke C, Hucko T, Manka R, Hamdan A, et al. Influence of left ventricular hypertrophy and geometry on diagnostic accuracy of wall motion and perfusion magnetic resonance during dobutamine stress. Circ Cardiovasc Imaging. 2010;3:507-14.

7. Herzog CA, Marwick TH, Pheley AM, White CW, Rao VK, Dick CD. Dobutamine stress echocardiography for the detection of significant coronary artery disease in renal transplant candidates. Am J Kidney Dis. 1999;33(6):1080-90.

8. Falcão SN, Rochitte CE, Junior WM, Quaglia L, Lemos PA, Sbano JC, et al. Incremental value of perfusion over wall-motion abnormalities with the use of dobutamine-atropine stress myocardial contrast echocardiography and magnetic resonance imaging for detecting coronary artery disease. Echocardiography. 2013;30(1):45-54

9. Tsutsui JM, Elhendy A, Anderson JR, Xie F, McGrain AC, Porter TR. Prognostic value of dobutamine stress myocardial contrast perfusion echocardiography. Circulation. 2005:112(10):1444-50.

10. Charoenpanichkit C, Morgan TM, Hamilton CA, Wallace EL, Robinson K, Ntim WO, et al. Left ventricular hypertrophy influences cardiac prognosis in patients undergoing dobutamine cardiac stress testing. Circ Cardiovascular Imaging. 2010;3(4):392-7.

11. Charoenpanichkit C, Little WC, Mandapaka S, Dall'Armellina E, Morgan TM, Hamilton CA, et al. Impaired left ventricular stroke volume reserve during clinical dobutamine stress predicts future episodes of pulmonary edema. J Am Coll Cardiol. 2011;57:839-48.

12. Natori S, Lai S, Finn JP, Gomes AS, Hundley WG, Jerosch-Herold M, et al. Cardiovascular function in multi-ethnic study of atherosclerosis: Normal values by age, sex, and ethnicity. AJR Am J Roentgenol. 2006;186:S357-65.

13. Chuang ML, Gona P, Hautvast GL, Salton CJ, Breeuwer M, O'Donnell CJ, et al. CMR reference values for left ventricular volumes, mass, and ejection fraction using computer-aided analysis: the Framingham Heart Study. J Magn Reson Imaging. 2014;39(4):895-900.17.

14. Dall'Armellina E, Morgan TM, Mandapaka S, Ntim W, Car JJ, Hamilton CA, et al. Prediction of cardiac events in patients with reduced left ventricular ejection fraction with dobutamine cardiovascular magnetic resonance assessment of wall motion score index. J Am Coll Cardiol. 2008;52(4):279-86.

15. Korosoglou G, Elhmidi Y, Steen H, Schellberg D, Riedle N, Ahrens J, et al. Prognostic value of high-dose dobutamine stress magnetic resonance imaging in 1,493 consecutive patients: Assessment of myocardial wall motion and perfusion. J Am Coll Cardiol. 2010;56(15):1225-34.

16. Gebker R, Jahnke C, Manka R, Hamdan A, Schnackenburg B, Fleck E, et al Additional value of myocardial perfusion imaging during dobutamine stress magnetic resonance for the assessment of coronary artery disease. Circ Cardiovasc Imaging. 2008;1(2):122-30.

17. Jahnke C, Nagel E, Gebker R, Kokocinski T, Kelle S, Manka R, et al. Prognostic value of cardiac magentic resonance stress tests: adenosine stress perfusion and docutamine stress wall motion imaging. Circulation. 2007;115(13):1769-76.

18. Drafts BC, Twomley KM, D'Agostin Jr R, Lawrence J, Avis N, Ellis LR, et al. Low to moderate dose anthracycline-based chemotherapy is associated with early noninvasice imaging evidence of subclinical cardiovascular disease. JACC Cardiovasc Imaging. 2013;6(8):877-85.

19. Nozawa T, Cheng CP, Noda T, Little WC. Effect of exercise on left ventricular mechanical efficiency in conscious dogs. Circulation. 1994;90:3047-54.

20. Vanoverschelde JL, Wijns W, Essamri B, Bol A, Robert A, Labar D, et al. Hemodynamic and mechanical determinants of myocardial o2 consumption in normal human heart: Effects of dobutamine. Am J Physiol. 1993;265:H1884-92.

21. Knaapen P, Germans T, Knuuti J, Paulus WJ, Dijkmans PA, Allaart CP, et al. Myocardial energetics and efficiency: Current status of the noninvasive approach. Circulation. 2007;115:918-27.

22. Nozawa T, Cheng CP, Noda T, Little WC. Relation between left ventricula oxygen consumption and pressure-volume area in conscious dogs. Circulation. 1994;89:810-7.

23. Kelly RP, Ting CT, Yang TM, Liu CP, Maughan WL, Chang MS, et al. Effective arterial elastance as index of arterial vascular load in humans. Circulation. 1992;86:513-21.

24. Cerqueira MD, Weissman NJ, Dilsizian V, Jacobs AK, Kaul S, Laskey WK, et al. Imaging AHAWGOMSaRfC. Standardized myocardial segmentation and nomenclature for tomographic imaging of the heart. A statement for healthcare professionals from the cardiac imaging committee of the council 
on clinical cardiology of the american heart association. Circulation. 2002;105:539-42

25. Redfield MM, Jacobsen SJ, Borlaug BA, Rodeheffer RJ, Kass DA. Age- and gender-related ventricular-vascular stiffening: a community-based study. Circulation. 2005;112(15):2254-62.

26. Geleijnse ML, Krenning BJ, van Dalen BM, Nemes A, Soliman OI, Bosch JG, et al. Factors affecting sensitivity and specificity of diagnostic testing: Dobutamine stress echocardiography. J Am Soc Echocardiogr. 2009;22:1199-208.

27. Gebker R, Frick M, Jahnke C, Berger A, Schneeweis C, Manka R, et al. Value of additional myocardial perfusion imaging during dobutamine stress magnetic resonance for the assessment of intermediate coronary artery disease. Int J Cardiovasc Imaging. 2012;28(1):89-97.

28. Cheng S, Fernandes VR, Bluemke DA, McClelland RL, Kronmal RA, Lima JA. Age-related left ventricular remodeling and associated risk for cardiovascular outcomes: the Multi-Ethnic Study of Atherosclerosis. Circ Cardiovascular Imaging. 2009;2(3):191-8

29. Borlaug BA, Lam CS, Roger VL, Rodeheffer RJ, Redfield MM. Contractility and ventricular systolic stiffening in hypertensive heart disease insights into the pathogenesis of heart failure with preserved ejection fraction. J Am Coll Cardiol. 2009;54(5):410-8.

30. Chantler PD, Nussbacher A, Gerstenblith G, Schulman SP, Becker LC Ferrucci $L$, et al. Abnormalities in arterial-ventricular coupling in older healthy persons are attenuated by sodium nitroprusside. Am J Physiol Heart Circ Physiol. 2011;300:H1914-22.

31. Vasu S, Morgan TM, Kitzman DW, Bertoni A, Stacey RB, Hamilton C, Chiles C, Thohan V, Hundley WG. Abnormal stress related measures of arterial stiffness in middle-aged and elderly men and women with impaired fasting glucose at risk for a first episode of symptomatic heart failure. J Am Heart Assoc. 2015 Jan 14;4(1). doi:10.1161/JAHA.114.00099.

32. Smart SC, Knickelbine T, Malik F, Sagar KB. Dobutamine-atropine stress echocardiography for the detection of coronary artery disease in patients with left ventricular hypertrophy. Importance of chamber size and systolic wall stress. Circulation. 2000;101:258-63.

33. Mirelis JG, Paetsch I, Jahnke C, Ibañez B, Fleck E, Alonso-Pulpon LA, et al. Impact of diastolic wall stress on the diagnostic value of visually interpreted dobutamine sress MR imaging. Int J Cardiol. 2014;172(1):e39-42.

34. Greenwood JP, Maredia N, Younger JF, Brown JM, Nixon J, Everett CC, et al. Cardiovascular magnetic resonance and single-photon emission computed tomography for diagnosis of coronary heart disease (ce-marc): A prospective trial. Lancet. 2012;379:453-60.

35. Schwitter J, Wacker CM, Wilke N, Al-Saadi N, Sauer E, Huettle K, et al. Mr-impact ii: Magnetic resonance imaging for myocardial perfusion assessment in coronary artery disease trial: Perfusion-cardiac magnetic resonance vs. Single-photon emission computed tomography for the detection of coronary artery disease: A comparative multicentre, multivendor trial. Eur Heart J. 2013;34:775-81.

\section{Submit your next manuscript to BioMed Central and take full advantage of:}

- Convenient online submission

- Thorough peer review

- No space constraints or color figure charges

- Immediate publication on acceptance

- Inclusion in PubMed, CAS, Scopus and Google Scholar

- Research which is freely available for redistribution 